\title{
Implementation of the expert decision system for environmental assessment in composite materials selection for automotive components
}

\begin{abstract}
Conventional materials selection system was replaced with sophisticated software tools by rapid changing technology. The growing environmental concerns and regulations widely among the industry, especially in automobiles, force us to explore the natural fiber materials as a replacement for synthetic materials which is in common use. As a result of extensive research and development, new natural fiber reinforced composite materials are emerging and the database of materials growing exponentially. The decision of selecting optimized materials was complicated, as it involves diversified choice of materials, coupled with various influencing criteria for the selection process. To abstain from deciding inappropriate materials, the technology of expert system software tools can help us in the appropriate materials selection. The objective of this research was to explore the implementation of Analytical Hierarchy Process (AHP) using the expert choice software tool for deciding optimum natural fiber reinforced composite materials by considering main criteria and subcriteria in the hierarchical model. The final judgement was performed with different scenarios of sensitivity analysis, giving priority to the environmental factors and sustainability. The result shows that the natural fiber composite material hemp and polypropylene gained the higher rank in the selection process and almost compliant with the requirements of industrial product design specification and can be recommended to automotive component manufacturers to enforce green technology.
\end{abstract}

Keyword: Expert system; Decision method; Materials selection; Analytical hierarchy process; Environmental factor; Natural fiber composites 